\title{
Impact of quarantine due to COVID infection on migraine: a survey in Genova, Italy
}

\author{
Italo Carlo Parodi ${ }^{1} \cdot$ Maria Gabriella Poeta $^{1} \cdot$ Andrea Assini $^{1} \cdot$ Erika Schirinzi $^{1,2}$ (I) $\cdot$ Paola Del Sette $^{3}$
}

Received: 22 May 2020 / Accepted: 21 June 2020 / Published online: 1 July 2020

(C) Fondazione Società Italiana di Neurologia 2020

\begin{abstract}
Quarantine is a well-known risk factor for psychological and psychiatric disturbances. We evaluated burden of migraine during lockdown due to COVID 19 pandemia. Forty-nine subjects followed in our headache clinic for migraine were evaluated for migraine burden by means of global assessment of migraine severity (GAMS) and visual analogue scale (VAS) by phone interview. Moreover, depression and anxiety were quantified by Beck depression inventory (BDI) and Zung Self-Rating Anxiety Scale (SAS). We evaluated changes in the value of migraine score from the 2 months immediately before lockdown (from January 1 to March 9) to the 2 months of quarantine (from March 10 to May 3). Value of GAMS was $5.61 \pm 0.76$ before and $4.16 \pm 1.46$ during quarantine $(p<.001)$. VAS was $7.49 \pm 1.10$ before and $5.47 \pm 1.88$ during quarantine $(p<.001)$. We also found a time by depression level interaction, $F(1,47)=6.21, p=.016, F(1,47)=14.52, p<.006$, respectively, showing that subjects with lower level of depression had better course of migraine. In conclusion, we showed that, during quarantine due to COVID pandemia, subjects with migraine had fewer migraine attacks and lesser pain and show moderate level of depression, correlated to migraine burden.
\end{abstract}

Keywords Covid $\cdot$ Quarantine $\cdot$ Migraine $\cdot$ Anxiety $\cdot$ Depression

\section{Introduction}

Quarantine is a well-known risk factor for psychological and psychiatric disturbances in hospital staff and in general population $[1,2]$.

Since late February 2020, a rapidly widespread rise of confirmed cases of novel coronavirus infection was observed in Italy; thus, a lockdown programme was started by the Government, from the beginning of March 2020. It established a period of travel ban and mandatory staying home (except for emergencies, health problems or regulated shopping only for bare necessities). The period lasted from March 10th until May 3rd.

Erika Schirinzi

e.schirinzi@gmail.com; erika.schirinzi@med.unipi.it

Neurology Unit, Galliera Hospital Genova, Genoa, Italy

2 Neurological Clinic, Department of Clinical and Experimental Medicine, University of Pisa, Via Roma 67, 56126 Pisa, Italy

3 Department of Brain and Behavioural Sciences, University of Pavia, Pavia, Italy
Migraine is associated with several comorbidities, among which anxiety and depression are by far the most frequent [3].

The aim of our study was to assess the impact of lockdown on migraine, in terms of migraine burden, but also on associated anxiety and depressive symptoms.

\section{Patients and method}

We selected a retrospective consecutive cohort of patients admitted to our Headache clinic from January 1 st to March 9th 2020 for the diagnosis of migraine. We excluded patients with first diagnosis of migraine, while considered only patients with follow-up visits, to avoid the effect of new therapies. We screened 76 patients. Then, we excluded patients with any change in prophylactic therapies $(N=10)$, patients at the first access $(N=13)$, and patients younger than 18 years $(N=4)$. The final sample consisted of 49 patients $(79.59 \% \mathrm{~F}$, mean age $=46.24 \pm 15.04$, age range $18-78$ years $)$. Of the overall sample, 10 patients had migraine with aura (20.4\%) and 39 patients had migraine without aura (79.6\%). In our clinic, all the subjects perform usually clinical evaluation, personal interview, evaluation of migraine severity by 
means of global assessment of migraine severity (GAMS) and visual analogue scale (VAS) [4, 5].

All the patients willing to participate in our survey, after informed consent, were subjected to standardized telephonic interview during which we collected data regarding migraine burden by means of GAMS and VAS, depression and anxiety by means of Beck depression inventory (BDI) and Zung SelfRating Anxiety Scale (SAS), respectively [4-7].

We also recorded the number of weekly usage of triptans pre- and during quarantine.

\section{Statistical analysis}

Paired sample $t$ test was performed to evaluate the changes in the value of migraine score from the 2 months immediately before lockdown (from January 1st to March 9th) to the 2 months of quarantine (from March 10th to May 3rd). We also evaluated presence or absence of anxiety or depressive disorders during quarantine.

We conducted mixed ANOVAs on migraine scores with time (before lock down, quarantine) as within-subject variable and depression level (minimal-mild, moderate-severe) as between-subject factor.

\section{Results}

Value of GAMS was $5.61 \pm 0.76$ before and $4.16 \pm 1.46$ during quarantine $(p<.001)$.

VAS was $7.49 \pm 1.10$ before and $5.47 \pm 1.88$ during quarantine $(p<.001)$.

Frequency of triptan utilization for migraine attacks (number pills/week) was $2.84 \pm 2.54$ in the period before as opposed to $2.00 \pm 1.78$ during quarantine $(p=.041)$ (Table 1$)$.

Value of Beck and Zung was, respectively, $13.10 \pm 7.33$ and $22.71 \pm 13.36$, indicating presence of mild to moderate level of depression and normal to mild level of anxiety. Given that only three patients showed mild to marked levels

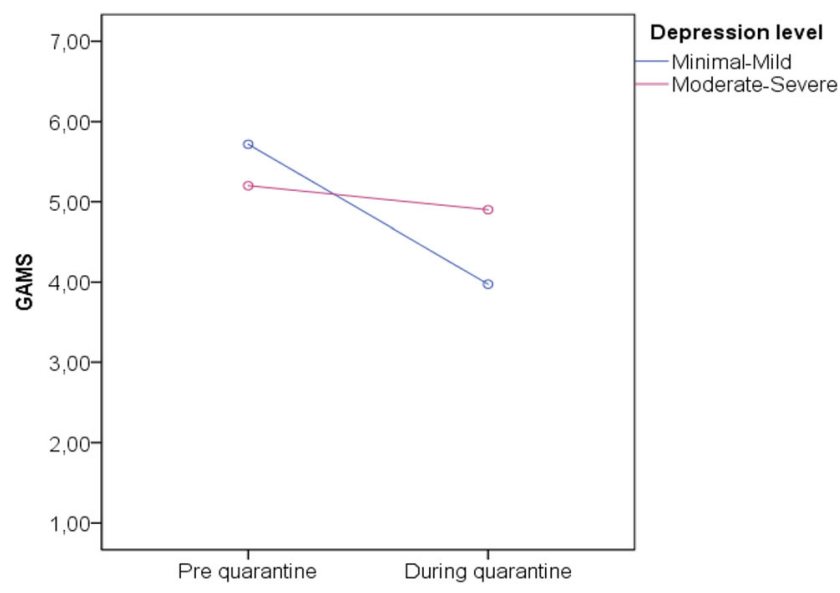

Fig. 1 Analysis of the interaction between depression (Beck) and reduction of migraine attack measured though GAMS. Red line subjects with minimal-mild level of depression. Blue line: subjects with moderate-severe level of depression

of anxiety during quarantine, we decided to focus the following analyses only on depression levels during quarantine.

The mixed ANOVAs on migraine severity, measured both with GAMS and VAS, showed a main effect on time, $F(1,47)=12.45, p=.001, F(1,47)=18.87, p<.001$, respectively, indicating an amelioration on migraine symptoms. Moreover, we also found a time by depression level interaction, $F(1,47)=6.21, p=.016, F(1,47)=14.52, p<.006$, respectively, showing that subjects with lower level of depression had better course of migraine (see Figs. 1 and 2).

\section{Discussion}

Our study showed that subjects with migraine had reduction of migraine evaluated by means of GAMS and VAS during quarantine. The trend of reduction of number of triptans per week is in line with this observation. The analysis of depression and anxiety showed mild-moderate level of depression and mild level of anxiety during the 2-month period of quarantine. No comparison was possible with the period before lockdown as we do not have the data.

Table 1 Migraine severity and frequency of triptan utilization for migraine attacks before and during quarantine

\begin{tabular}{|c|c|c|c|c|c|c|}
\hline & \multirow[t]{2}{*}{ Mean (sd) } & \multirow[t]{2}{*}{ Possible range } & \multirow[t]{2}{*}{$t(d f=48)$} & \multicolumn{2}{|l|}{$95 \% \mathrm{CI}$} & \multirow[t]{2}{*}{$p$} \\
\hline & & & & Lower & Upper & \\
\hline GAMS pre-quarantine & $5.61(.76)$ & $1-7$ & 5.90 & .95 & 1.93 & .000 \\
\hline GAMS during quarantine & $4.16(1.46)$ & & & & & \\
\hline VAS pre-quarantine & $7.49(1.10)$ & $0-10$ & 6.98 & 1.44 & 2.60 & .000 \\
\hline VAS during quarantine & $5.47(1.88)$ & & & & & \\
\hline Number pills/week pre-quarantine & $2.84(2.54)$ & & 2.10 & .036 & 1.64 & .041 \\
\hline Number pills/week during quarantine & $2.00(1.78)$ & & & & & \\
\hline
\end{tabular}




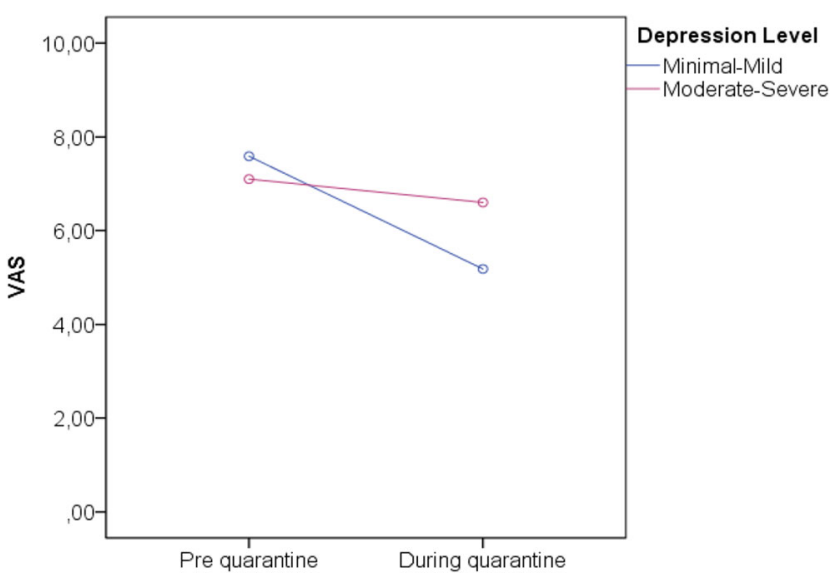

Fig. 2 Analysis of the interaction between depression (Beck) and reduction of migraine attack measured though VAS. Red line subjects with minimal-mild level of depression. Blue line: subjects with moderatesevere level of depression

A possible interpretation of result might be that being at home, with relative reduction of stressor coming from working activity, may cause a reduction of triggers for migraine attacks. Presence of depression and anxiety could be related to the specific causes of quarantine, determined by Covid pandemia and lockdown. Moreover, there was an interaction between depression and migraine burden, with better course of migraine in less depressed subjects.

Limitations of our study are the retrospective design and the lack of analysis of psychiatric comorbidities before the quarantine period. Indeed, we did a pre-post analysis, subjected to several biases; yet, we considered the variable "COVID quarantine" as a whole, without analytical evaluation of single possible causes of changes in migraine burden.

Strength of our study is the consecutive recruitment, the few number of lost to follow-up, the utilization of specific validated tests for migraine and pain and for psychiatric comorbidities.

In conclusion, we showed that, during quarantine due to COVID pandemia, subjects with migraine had fewer migraine attacks and lesser pain, despite moderate level of depression.

\section{Compliance with ethical standards}

Conflict of interest The authors declare that they have no conflict of interest.

Informed consent The research involves patients who gave informed consent for publications.

Ethical approval None.

\section{References}

1. Kisely S, Warren N, McMahon L et al (2020) Occurrence, prevention, and management of the psychological effects of emerging virus outbreaks on healthcare workers: rapid review and meta-analysis. BMJ. 369:m1642

2. Qiu J, Shen B, Zhao M, Wang Z, Xie B, Xu Y (2020) A nationwide survey of psychological distress among Chinese people in the COVID-19 epidemic: implications and policy recommendations. Gen Psychiatr 33(2):e100213

3. Buse DC, Reed ML, Fanning KM, Bostic R, Dodick DW, Schwedt TJ, Munjal S, Singh P, Lipton RB (2020) Comorbid and cooccurring conditions in migraine and associated risk of increasing headache pain intensity and headache frequency: results of the migraine in America symptoms and treatment (MAST) study. J Headache Pain 21(1):23

4. Sajobi TT, Amoozegar F, Wang M, Wiebe N, Fiest KM, Patten SB, Jette N (2019) Global assessment of migraine severity measure: preliminary evidence of construct validity. BMC Neurol 19(1):53

5. Karcioglu O, Topacoglu H, Dikme O, Dikme O (2018) A systematic review of the pain scales in adults: which to use? Am J Emerg Med 36(4):707-714

6. Richter P, Werner J, Heerlein A, Kraus A, Sauer H (1998) On the validity of the Beck depression inventory. A review. Psychopathology 31(3):160-168 Review

7. Dunstan DA, Scott N, Todd AK (2017) Screening for anxiety and depression: reassessing the utility of the Zung scales. BMC Psychiatry 17(1):329

Publisher's note Springer Nature remains neutral with regard to jurisdictional claims in published maps and institutional affiliations. 